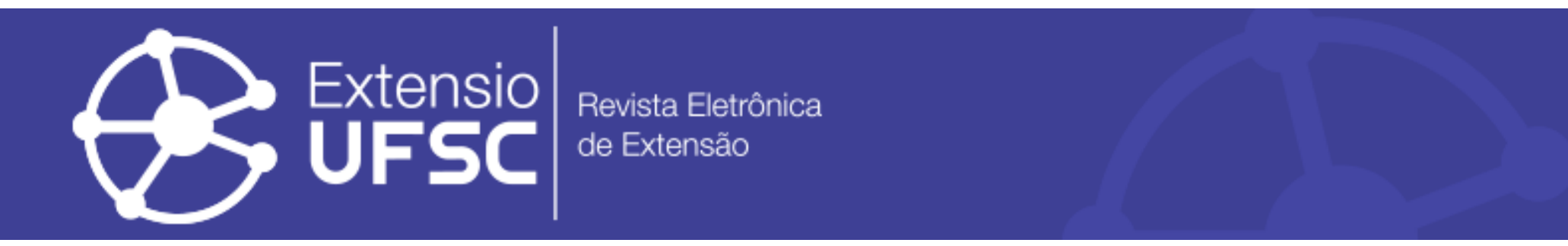

\title{
VER-SUS: PERCEPÇÕES DOS VIVENTES VOLTADAS AO CONTEXTO UNIVERSITÁRIO E SOCIAL
}

\author{
Aline Santana Figueredo \\ Universidade Federal do Maranhão \\ aline_ibms@hotmail.com \\ Aldo Lopes da Costa Júnior \\ Universidade Federal do Maranhão \\ jrlopesc@gmail.com \\ Gustavo de Almeida Santos \\ Universidade Federal do Maranhão \\ gustavoalmeidaitz@gmail.com
}

\author{
Francisco Eduardo Ramos da Silva \\ Faculdade de Ciências e Tecnologia do Maranhão \\ eduardocx85@gmail.com \\ Vitor Pachelle Lima Abreu \\ Universidade Federal do Maranhão \\ vpachelle@gmail.com \\ Wherveson de Araújo Ramos \\ Unidade de Ensino Superior do Sul do Maranhão \\ wherveson@hotmail.com
}

\section{Resumo}

O objetivo do artigo é analisar a percepção dos participantes da primeira edição do VER-SUS Marabá-PA, no que tange ao contexto universitário e social do projeto. Trata-se de um estudo do tipo descritivo e exploratório, com abordagem qualitativa dos dados. A coleta de dados foi realizada nos meses de janeiro e fevereiro pela ferramenta Google Docs. A análise de dados foi feita através do método de análise qualitativa de Minayo. A partir da análise de dados, emergiram as seguintes categorias: visão pré-vivência; o que penso do SUS de Marabá; impressão sobre as comunidades específicas; VER-SUS como articulador para as ações sociais; e visão pós-vivência. A participação no projeto VER-SUS proporciona quebra de paradigmas e do pensar acadêmico voltado unicamente ao curso, promovendo um olhar para além dos muros da universidade, instigando o questionamento em relação às interações sociais e à maneira de como se faz saúde pautada no Sistema Único de Saúde.

Palavras-chave: Saúde Pública. Acadêmicos. Estágio de Vivência.

\section{VER-SUS: PERCEPTIONS OF THE VIVENTS RETURNED TO THE UNIVERSITY AND SOCIAL CONTEXT}

Abstract

The objective of the article is to analyze the perception of the participants of the first edition of the VER-SUS Marabá, regarding the university and social context of the project. It is a descriptive / exploratory study, with a qualitative approach to the data. The data collection was carried out in the months of January and February in a technological tool called Google Docs. Data analysis was done using Minayo's qualitative analysis method. From the data analysis, the following categories emerged: pre-living vision; What do I think of the SUS of Marabá? Printing on specific communities; SEE-SUS as an articulator for social actions; Post-living vision. Participation in the project VER-SUS provides a breakdown of paradigms and academic thinking focused solely on the course, promoting a look beyond the walls of the university, instigating the questioning of social interactions and the way health is based on the Unified Health System.

Keywords: Public health. Academics. Internship.

\section{VER-SUS: PERCEPCIONES DE LOS VIVIENTES VOLTADAS AL CONTEXTO UNIVERSITARIO Y SOCIAL}

\section{Resumen}

El objetivo del artículo es analizar la percepción de los participantes de la primera edición de VER-SUS Marabá, sobre el contexto universitario y social del proyecto. Es un estudio descriptivo / exploratorio, con un enfoque cualitativo de los datos. La recolección de datos se llevó a cabo en los meses de enero y febrero en una herramienta tecnológica llamada Google Docs. El análisis de los datos se realizó utilizando el método de análisis cualitativo de Minayo. Del análisis de datos surgieron las siguientes categorías: visión pre-viviente; ¿Qué pienso del SUS de Marabá? Impresión en comunidades específicas; VER-SUS como articulador de acciones sociales; Visión post-vida. La participación en el proyecto VER-SUS proporciona un desglose de los paradigmas y el pensamiento académico centrado exclusivamente en el curso, promoviendo una mirada más allá de las paredes de la universidad, promoviendo el cuestionamiento de las interacciones sociales y la forma en que la salud se basa en el Sistema Único de Salud.

Palavras clave: Salud pública. Académicos. Etapa de Vivencia. 
VER-SUS: percepções dos viventes voltadas ao contexto universitário e social

\section{INTRODUÇÃO}

A construção do Projeto Vivências e Estágios na Realidade do SUS (VER-SUS/Brasil) foi influenciada por movimentos sociais e diversos projetos realizados anteriormente, como: estágios interdisciplinares de vivência em assentamentos rurais, organizados pela Federação dos Estudantes de Agronomia do Brasil (FEAB) no final da década de 1980; projetos de estágio de vivência realizados pela Direção Executiva Nacional dos Estudantes de Medicina (DENEM) nos anos 1990, e o projeto Escola de Verão, realizado com o apoio da Escola de Saúde Pública do Estado do Rio Grande do Sul (ESP/RS), em 2002 (CECCIM \& BILIBIO, 2004).

Os estágios de vivências constituem, portanto, importantes dispositivos que permitem ao estudante experimentar um novo espaço de aprendizagem que é o cotidiano de trabalho das organizações de saúde, entendido enquanto princípio educativo e espaço de desenvolvimento de processos de luta dos setores do campo da saúde, possibilitando a formação de profissionais comprometidos ética e politicamente com as necessidades de saúde da população (BRASIL, 2013).

Sendo assim, compreende-se que o VER-SUS permite aos participantes experiências e vivências no cotidiano dos trabalhos de organizações e serviços de saúde, mostrando as diversas realidades dos setores de serviço, isto tudo com a proposta de estimular a formação de profissionais que trabalhem no SUS e que lutem por ele, de forma comprometida, ética e politicamente com as necessidades da população.

A partir disso, o presente estudo tem como objetivo analisar a percepção dos participantes da primeira edição do VER-SUS Marabá, no que tange ao contexto universitário e social do projeto.

\section{MATERIAIS E MÉTODOS}

Trata-se de um estudo do tipo descritivo e exploratório, com abordagem qualitativa dos dados. Estudos exploratórios são investigações de pesquisa empírica, cujo objetivo é a formulação de questões ou de um problema; emprega-se geralmente procedimentos sistemáticos ou para a obtenção de observações empíricas ou para as análises de dados (ou ambas, simultaneamente). Obtêm-se frequentemente descrições tanto quantitativas quanto qualitativas do objeto de estudo, e o investigador deve conceituar as inter-relações entre as propriedades do fenômeno, fato ou ambiente observado (LAKATOS, 2003). 
Contudo, o presente estudo contou com a abordagem qualitativa, propondo buscar uma interdependência viva entre o sujeito e o objeto, interagindo com a dinâmica do mundo real, procurando entender a realidade de um determinado fenômeno social, tendo o pesquisador como principal instrumento para aquisição de informações, que posteriormente poderão também ser representadas de forma mais ampla (MINAYO\&GUERRIERO, 2014).

A coleta de dados foi realizada nos meses de janeiro e fevereiro de 2018. Os participantes foram contatados previamente por uma rede social, sendo feito o convite e esclarecido a finalidade da pesquisa. Depois de informados, receberam o link de questionário semiestruturado, contendo perguntas acerca do perfil desses indivíduos e também perguntas subjetivas sobre a visão dos participantes acerca da vivência, disposto na ferramenta Google Docs. De acordo com a Resolução 510/2016, seção II, art 17 \$2 $2^{\circ}$, no caso desse tipo de registro não ser assinado por escrito, o participante poderá ter acesso ao termo de consentimento livre esclarecido (TCLE). Este modelo de coleta de dados pode ser classificado por Mattar (2008), como questionário autopreenchido, no qual o entrevistador não irá praticar nenhum tipo de intervenção durante a pesquisa.

As informações coletadas foram analisadas através da técnica de análise de conteúdo, na modalidade temática, tomando como base os temas abordados nos relatos dos informantes (MINAYO, 2002).

A análise de dados se deu em três momentos: pré-análise (etapa de organização do material analisado com leitura flutuante dos enunciados sob a orientação das regras de exaustividade, representatividade, homogeneidade e pertinência, para a constituição do corpus); exploração do material (seleção das falas dos informantes e organização das categorias ou núcleos temáticos); e interpretação dos resultados (submissão do material explorado a um tratamento interpretativo e contextualizado). Sendo assim, após a leitura flutuante do material se deu a constituição do corpus, com base nos critérios de exaustividade, representatividade e pertinência (MINAYO, 2002).

A partir da análise de dados, emergiram as seguintes categorias: visão pré-vivência; o que penso do SUS de Marabá; impressão sobre as comunidades específicas; VER-SUS como articulador para as ações sociais; e visão pós-vivência.

No que se referem aos preceitos éticos, foram aplicados todos os procedimentos éticos presente na resolução 466/2012 do Conselho Nacional de Saúde (CNS), que trata da pesquisa envolvendo seres humanos, sendo submetido para apreciação ao comitê de ética em pesquisa da Universidade Federal do Maranhão -UFMA, recebido o parecer aprovado, com n ${ }^{\circ} 2.386 .300$. 
VER-SUS: percepções dos viventes voltadas ao contexto universitário e social

Além disso, foram aplicados Termos de Consentimento Livre e Esclarecido com devida autorização das pessoas que responderam aos questionários voluntariamente.

\section{RESULTADOS E ANÁLISES}

A primeira edição do projeto Vivências e Estágios na Realidade do Sistema Único de Saúde na cidade de Marabá-PA ocorreu no primeiro semestre de 2018, e contou com a participação de 36 estudantes de graduação, sendo facilitadores e viventes.

Participaram da pesquisa 15 acadêmicos de graduação, residentes de cinco estados do território brasileiro: Maranhão, Pará, Tocantins, Ceará e Piauí. Dos entrevistados, 11 eram do sexo feminino, possuíam idade entre 19 e 34 anos, nove dos entrevistados afirmaram residir no estado do Pará, sendo que seis são da cidade de Marabá. Quanto à instituição de ensino superior, todos afirmaram estudar em universidades públicas, sendo que seis estudam na Universidade Federal do Sul e Sudeste do Pará (UNIFESSPA) .Ainda questionados sobre o curso de graduação, a grande maioria afirmou que faz o curso de bacharelado em Enfermagem e estão nos primeiros períodos da graduação.

Para as questões subjetivas, usou-se a técnica de análise de conteúdo, de onde emergiram as categorias: visão pré-vivência; o que penso do SUS de Marabá; impressão sobre as comunidades específicas e VER-SUS como articulador para as ações sociais.

\section{Categoria 1: Visão pré-vivência}

Nesta categoria são expressas em falas, a visão dos participantes acerca da vivência em Marabá, tendo sido questionado aos participantes quais expectativas tinham em relação à vivência na cidade, e que foram expressadas nas seguinte falas:

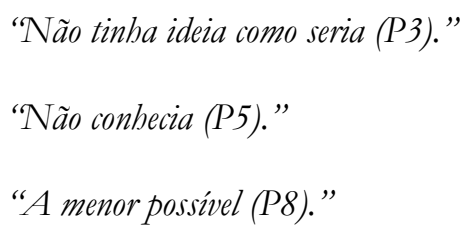

O contato proporcionado pela imersão possibilita ao estudante conhecer como estão estruturados e organizados os serviços de saúde através do contato real com a dinâmica de funcionamento do SUS. Através desse contato, o estudante desenvolve um senso reflexivo-crítico mais apurado dando os subsídios necessários para uma formação técnica, científica, cidadã e 
VER-SUS: percepções dos viventes voltadas ao contexto universitário e social

política. Diante disso, enfatiza-se que o processo vivenciado ratificou o quanto são importantes as ações dos acadêmicos, enquanto protagonistas na construção de um SUS no qual suas diretrizes e regulamentações sejam realmente concretizadas, pois a consolidação do sistema passa por conquistas e desafios que requerem uma conduta diferenciada (FERREIRA et al, 2016).

Através dos relatos, também se pode perceber que alguns dos viventes tinham pouca noção em relação ao projeto em si, pois não possuíam nenhum conhecimento ou eram limitados. Em outras falas, nota-se que outros participantes estavam com muitas expectativas e sabiam que o estágio iria mudar sua percepção em relação ao ambiente da saúde em diversas formas.

\section{"Era algo desconhecido, havia muita curiosidade e expectativa, não tinha idéia de como seria, apenas sabia que seria algo agregador (P9)." \\ "Uma oportunidade valiosa de poder vivenciar o SUS além do que a Universidade poderia ofertar (P15).”}

Com base nas falas, percebe-se que as instituições de ensino superior não formam graduandos para compreender o SUS, utilizam ainda o sistema de aprendizado técnico-científico, deixando de lado, a atuação mais crítica e reflexiva dentro do sistema (CANÔNICO; BRETAS 2008).

Os currículos dos cursos sempre privilegiaram o paradigma curativo, e essa continua sendo a filosofia de muitas universidades, cujo ensino não se orienta pela situação epidemiológica social, cultural e econômica da população, ou seja, priorizando os problemas de saúde individuais e não o coletivo (MENEZES et al, 2012).

O SUS não deve ser simplesmente um campo de estágio para aprendizagem prática, mas sim um interlocutor das instituições de ensino a partir de questões relacionadas à gestão dos serviços, assim estabelecendo elaboração e implantando políticas vinculadas à formação profissional (NUNES, 2006).

Backes et al, (2014), afirma que, o domínio do conhecimento técnico é indispensável, porém não é suficiente para a construção da relação teórica e prática no campo da saúde para além da formação específica, sendo assim, espera-se que o profissional com formação de saúde compreenda de forma crítica e reflexiva as políticas que norteiam o campo da saúde (SPERANDIO; TORRES, 2016).

Sendo assim, o VER-SUS provoca os acadêmicos para que eles reflitam sobre a sua formação e as necessidades de mudanças no atendimento realizado no SUS, buscando formar profissionais mais favoráveis ao trabalho em equipe, aos trabalhos preventivos e de promoção em saúde, com um foco maior nas necessidades dos indivíduos. A interação com outros estudantes 
VER-SUS: percepções dos viventes voltadas ao contexto universitário e social

proporciona a troca de conhecimentos, a cooperação entre os futuros profissionais da saúde e a conscientização dos deveres de cada profissão. Além do que, o trabalho em equipe interprofissional minimiza a sobrecarga de algumas profissões (FERREIRA et al, 2016).

\section{Categoria 2: o que penso do SUS de Marabá?}

$\mathrm{Na}$ segunda categoria é mostrada através de relatos a impressão dos viventes acerca das comunidades específicas. O primeiro questionamento foi voltado para o Movimento dos Trabalhadores Sem Terra (MST), em que foi perguntado sobre a impressão em relação ao movimento e aos fatos mais marcantes.

\footnotetext{
"A impressão que tive foi sobre pessoas batalbadoras que reivindicam pelos seus direitos, o que me marcou foi a falta do acesso à saúde, uma vez que eles não possuem [...] (P3)."

"São pessoas admiráveis que se impõem perante a sociedade, buscando lutar pelos seus direitos; a determinação deles perante as dificuldades enfrentadas [...]No entanto me trouxe o sentimento de indignação devido à falta de serviço de saúde para atendê-los próximo ao local onde se encontram" (P7)."
}

Ao serem feitos os mesmos questionamentos, mas voltados para a população indígena, surgiram as seguintes respostas:

\footnotetext{
"A resistência das pessoas mesmo com todos os problemas que enfrentam apenas para sobreviver. Todos os conflitos com empresas que destroem suas terras e sua cultura. Como a aproximaşão com o estilo de vida do homem branco prejudicou a saúde dessa populaşão, que passou a sofrer de doenças que não sofriam, além dos problemas de exclusão social e da perda de identidade que alguns sofrem (P14)."

"A saúde não está chegando nas aldeias e quando chega é uma situação precária, pois só vem um funcionário da saúde uma vezpor mês e não cobre todos os cidadãos (P12)."
}

Portanto, as vivências necessitam de um alinhamento quanto à integralidade e equidade, ampliando o conceito saúde valorizando todos os sujeitos e seus ambientes nos quais estão inseridos, com potencialidade para transformar os modelos de gestão, práticas e atenção em saúde (FERLA et al., 2013).

Nessa perspectiva, as visitas nas comunidades é uma importante vivência dentro do projeto, por colocar os participantes em confronto com os próprios preconceitos adquiridos ao longo da sua formação enquanto indivíduo, descontruindo os existentes e reconstruindo-os, assim, haverá um reconhecimento relacionado ao fazer saúde daquela comunidade, fortalecendo o respeito à cultura e religiosidade da população envolvida. 
VER-SUS: percepções dos viventes voltadas ao contexto universitário e social

Diante disso, Moraes e Batiston (2016), afirmam que, para uma assistência em saúde mais íntegra é necessário que se exerçam as diretrizes que o SUS preconiza, como um atendimento que vise à universalidade, equidade e à integralidade. Contudo, quando se observa a real situação do país se percebe alguns déficits, os quais devem ser trabalhados e isso ocorrerá com as lutas sociais, os usuários, os profissionais da saúde e os gestores. O VER-SUS apresenta-se como possibilidade de transformação de um sistema formativo engessado, verticalizado.

O VER-SUS também permite que os acadêmicos percebam o contraste entre teoriaprática, que na maioria das vezes, infelizmente, não corresponde à realidade e demanda social, sendo assim, necessita de adequações para sua efetivação.

\section{Categoria 3: Impressão sobre as comunidades específicas e o VER-SUS como articulador para as ações sociais.}

A terceira categoria se refere à influência do VER-SUS Marabá nos projetos sociais, em que o questionamento foi voltado para a ótica dos participantes em relação aos espaços de ações sociais. Através da visita a esses locais, os estudantes puderam ver o caráter humanizador e serem imersos nessa realidade, realidade essa que muitos não têm a oportunidade de vivenciar durante a vida acadêmica. Seguem os relatos:

"No geral, o que mais marcon foi perceber como a sociedade de certa forma se organiza para suprir certas demandas no município. Muito bom também tentar refletir sobre como estes locais podem acudir necessidades sociais na região (P4)".

"Que são projetos maravilhosos e inclusive deveria exigir mais projetos do tipo, o que me marcou foi o trabalho com dedicaşão sem medição de esforço para ajudar o próximo (P8). "

Esta experiência foi crucial para aguçar a nossa criticidade no processo formativo e na futura atuação enquanto profissional incitando a proatividade e proficiência em prol do SUS. A vivência foi uma oportunidade de intercâmbio entre diversos saberes, discussões sobre a conjuntura política do SUS e o estímulo para que busquemos os subsídios necessários para provocar mudanças que contribuam de forma positiva para o processo formativo e a sociedade.

Este tipo de contato estimula o trabalho em equipe, desenvolvimento e aperfeiçoamento de habilidades e competências possibilitando a relação de interdisciplinaridade dos envolvidos ampliando a concepção de saúde e mostrando que todos possuem um papel de extrema importância na consolidação do SUS. Na área da saúde, o trabalho em equipe tem o objetivo de compartilhar o planejamento, cooperar, colaborar e interagir, inserindo os atores, saberes, práticas, interesses e necessidades (RIBEIRO, PIRES, BLANK, 2003). 
VER-SUS: percepções dos viventes voltadas ao contexto universitário e social

Além disso, é importante testificar que a experiência não se reflete apenas na vida acadêmica, mas no processo profissional dos viventes, gerando novas dimensões de aprendizado e experiência sobre o trabalho em equipe bem como a didática abordada em grupos, moldando por tanto o trabalho de acordo com as necessidades do grupo.

Relacionar-se com os outros viventes possibilitou a percepção de que os conhecimentos entre as diversas áreas do saber contribuem de forma significativa para a compreensão da conjuntura social-política incitando uma reflexão conjunta que proporcione uma visão holística e integral do indivíduo aumentando o ensejo por um processo formativo intenso e dinâmico pautado nos princípios éticos, morais e científico.

\section{CONSIDERAÇÕES FINAIS}

A participação no projeto VER-SUS proporciona quebra de paradigmas e do pensar acadêmico voltado unicamente ao curso, promovendo um olhar para além dos muros da universidade, instigando o questionamento em relação às interações sociais e à maneira como se faz saúde pautada no Sistema Único de Saúde. Contemplando ainda a contribuição do aprendizado nos diferentes aspectos do processo formativo, as esferas política, social, técnica, intelectual e científica pautada em princípios éticos e morais estimulando a formação de profissionais aptos.

Permite, ainda, a realização de visitas a espaços sociais e estabelecimentos de saúde, proporcionando o conhecimento acerca da forma como cada comunidade faz saúde, também possibilitou aos estudantes conhecerem a realidade do SUS de Imperatriz e poderem comparar com a de seus estados. Frente a isso, tal projeto, seguindo os traços da epistemologia freiriana direciona o entendimento da dimensão dos sentidos, e propõe pensar a realidade na qual nos inserimos, compreendendo que o pensamento é conduzido conforme a situações concretas que visualizamos.

Portanto, proporcionar vivências nesse âmbito é ativar e promover novas medidas complementares de aprendizado que se respaldam numa realidade atual do SUS. Na medida que o indivíduo é inserido na experiência ele é direcionado para uma formação acadêmica diferenciada com novas buscas do conhecimento, preparo profissional, engajamento e envolvimento embasado na reflexão, criticidade e ainda na compreensão da conjuntura do SUS. 
VER-SUS: percepções dos viventes voltadas ao contexto universitário e social

\section{REFERÊNCIAS}

ATAIDES, R.J.C., BARBOSA, R. L.; RAMOS, T. L.; NETO,A. R. S.; SILVA, F. E. R.; ABREU, V. P. L. A implicação do estágio e vivência na realidade do Sistema Único de Saúde do Maranhão para a formação de futuros profissionais humanizados: um relato de experiência. In: $12^{\circ}$ Congresso Internacional da Rede Unida, 1, 2016. Anais. Campo Grande: Rede Unida, 2016. p. 193-276.

BACKES, D.S. et al. O Sistema Único de Saúde idealizado versus o realizado: contribuições da Enfermagem. Rev. Latino-Am. Enfermagem. v. 22, n.6, p.1026-1033, 2014.

BRASIL. Ministério da Saúde. Secretaria Executiva. Sistema Único de Saúde (SUS): princípios e conquistas. Brasília: Ministério da Saúde; 2013.

CANÔNICO RP, BRÊTAS ACP. Significado do programa vivência e estágios na realidade do Sistema Único de Saúde para formação profissional na área da saúde. Acta Paul Enferm [Internet]. 2008. Disponível em: http://www.scielo.br/pdf/ape/v21n2/a04v21n2.pdf .

CECCIM, R.B; Feuerwerker, L.C.M.. Quadrilátero da formação para a área da saúde: ensino, gestão, atenção e controle social PHYSIS: Rev. Saúde Coletiva, Rio de Janeiro, v.14, n.1, p.4165, 2004.

CECCIM, R. B., \& BILIBIO, L. F. S. Articulação com osegmento estudantil da área de saúde: uma estratégia de inovação na formação de recursos humanos para o SUS. In Ministério da Saúde (Org.), VER-SUS/BRASIL: Cadernos deTextos(p. 4-19). Brasília, DF: (2004).

LAKATOS, Eva Maria. Fundamentos de metodologia. 5. ed. - São Paulo : Atlas 2003.

MENDES FMS, FONSECA KA, BRASIL JA, DALBELLO-ARAÚJO M. Ver-Sus: Relato de Vivências na Formação de Psicologia. PsicolCiêncProf [Internet]. 2012. Disponível em: http://www.scielo.br/pdf/pcp/v32n1/v32n1a13.pdf .

MINAYO, Maria Cecília de Souza. Pesquisa social: teoria, método e criatividade. 21 ed. Rio de Janeiro: Editora Vozes, 2002.

MINAYO, MCS; GUERRIERO ICZ. Reflexividade como éthos da pesquisa qualitativa. Ciência \& Saúde Coletiva, 19(4):1103-1112, 2014. Disponível em : http://www.scielo.br/pdf/csc/v19n4/1413-8123-csc-19-04-01103. Acesso em 25 de Abril de 2017.

NUNES, E.D. Saúde coletiva: uma história recente de um passado remoto. In: Campos GWS, et al (editores). Tratado de saúde coletiva. 2a ed. São Paulo: Hucitec; p.19-40, 2006.

Recebido em: 26/06/2018

Aceito em: 30/07/2019 